\title{
Análise de questões do SAERS e o ensino de álgebra na perspectiva dos registros de representação
}

\author{
Analysis of SAERS issues and teaching algebra in the perspective o \\ representative registers
}

\author{
Raquel Taís Breunig \\ raqueltaisb@yahoo.com.br
}

Cátia Maria Nehring

catia@unijui.edu.br

\begin{abstract}
Resumo
O presente trabalho se deu a partir da elaboração e desenvolvimento do projeto de pesquisa - Análise de Questões do SAERS e o Ensino de Álgebra na Perspectiva dos Registros de Representação, desenvolvido no curso de Matemática de uma universidade particular do Rio Grande do Sul. A Teoria dos Registros de Representação Semiótica (DUVAL, 2003) subsidiou a pesquisa, juntamente com as Dimensões da Álgebra propostas pelos Parâmetros Curriculares Nacionais - Matemática (BRASIL, 1998), o Referencial Curricular do Rio Grande do Sul (2009), os Boletins Pedagógicos de Avaliação da Educação (RS, 2007 e 2008) e as ideias da álgebra de Coxford e Shulte (1995). A partir deste estudo teórico, foram analisados os itens disponíveis da avaliação do SAERS, dos períodos de dois mil e sete, dois mil e oito e dois mil e nove, aplicadas a alunos do primeiro ano do Ensino Médio, considerando os Registros de Representação Algébricos e suas transformações. Sequencialmente, foram selecionados três itens, um de cada período, nos quais pode-se identificar, a partir do estudo teórico, maior possibilidade de mobilizar os Registros Algébricos. Por meio desta análise foi possível constatar que é dada grande ênfase às operações com números naturais em situações problema, ou seja, o Registro Numérico e o tratamento deste é predominante, consequentemente, há pouca ênfase em relação aos conceitos e Registros Algébricos, bem como, à conversão entre eles.
\end{abstract}

Palavras-chave: Registros de Representação Semiótica. Ensino de Álgebra. SAERS. Avaliação Pública.

\begin{abstract}
This study took place from the establishment and development of the research project - Analysis of SAERS Issues and Teaching Algebra in the Perspective o Representative Registers, developed as a criterion to conclude the Degree in Mathematics in a private University of Rio Grande do Sul. The theory of semiotic representation registers (Duval, 2003), funded the research along with the dimensions of algebra proposed by the National Curricular Parameters - Mathematics (BRAZIL, 1998), the Curricular Reference of Rio Grande do Sul (2009) the Pedagogical report cards of Education Evaluation (RS, 2007 and 2008) and the ideas of algebra from Coxford and Shulte (1995). From this theoretical study, the available items of SAERS assessing were analyzed, from the periods of two thousand and seven, two thousand and eight and two thousand and nine, the assessment was applied to first year students in high school, considering the Algebraic Representation Registers and their changes. Sequentially, three items were selected, from them, one of each period, in which were possible to identify, from the theoretical study, a greater possibility of mobilizing the Algebraic Registers. From the
\end{abstract}


analysis, wit was determined that a great emphasis is given to operations with natural numbers in problem situations, it mean that the registration number and its treatment is prevalent, therefore, there is a small emphasis in relation to algebraic concepts and registers, as well as conversion between them.

Keywords: Registers of semiotic representation. The Algebra Teaching. SAERS. Public Assessment.

\section{Introdução}

Uma das atuais preocupações relacionadas à educação é a qualidade do ensino nas escolas. Para tanto, foram criados sistemas de avaliação que analisam a qualidade do ensino, considerando o desempenho dos alunos e a capacidade de desenvolver habilidades e competências. Entende-se a avaliação como parte do processo de ensino e aprendizagem, com o intuito de determinar se os objetivos do ensino estão sendo alcançados (CAMPOS et al., 2003 apud ALMOULOUD, 2007).

Em pesquisas realizadas por Breunig, Nehring e Pozzobon (2010), foi possível identificar as dificuldades relatadas e observadas por professores e alunos, no ensinar e aprender, no que tange o Ensino de Álgebra. É possível, também, perceber grande dificuldade por parte dos alunos com relação a identificação e mobilização de Registros Algébricos. Tais afirmações possibilitam identificar a importância de se realizar pesquisas e discussões acerca do ensino e aprendizagem de conceitos algébricos.

Diante desses fatos busca-se, neste artigo, analisar os itens que compõem uma das diversas avaliações públicas, enfatizando os conceitos algébricos, a Teoria dos Registros de Representação Semiótica (DUVAL, 2003) e os documentos oficiais de ensino. Procura-se identificar os Registros de Representação Algébricos mobilizados e os mais enfatizados, considerando os itens que possibilitam a mobilização dos Registros de Representação Algébricos nas avaliações do Sistema de Avaliação do Rendimento Escolar do Rio Grande do Sul (SAERS), aplicadas nos períodos de dois mil e sete, dois mil e oito e dois mil e nove, a alunos do primeiro ano do Ensino Médio. Tal análise mostra-se relevante, pois é importante identificar o que está sendo exigido em relação ao Ensino de Matemática no Brasil, contribuindo para uma reflexão em busca de melhores estratégias de ensino e aprendizagem, especificamente em relação ao ensino conceitual de Álgebra. 


\section{Procedimentos Metodológicos}

Por meio de uma abordagem qualitativa, realizou-se um estudo teórico dos Registros de Representação Semiótica, propostos por Duval (2003), na qual pode-se identificar a importância destes no ensinar e aprender Matemática, considerando especificamente os conceitos algébricos. Além disso, foi realizado um estudo dos documentos oficiais, no qual foi possível identificar a importância de o aluno compreender significativamente os conceitos algébricos, pois o Referencial Curricular do Rio Grande do Sul (RS, 2009), os Parâmetros Curriculares Nacionais - Matemática (BRASIL, 1998) e os autores Coxford e Shulte (1995), enfatizam a importância do aluno reconhecer a Álgebra em suas diferentes concepções, ou seja, as dimensões da Álgebra.

Este estudo teórico subsidiou a análise dos itens disponibilizados nos Boletins Pedagógicos de Avaliação da Educação do SAERS, do primeiro ano do Ensino Médio, aplicadas em várias escolas públicas e privadas do estado do Rio Grande do Sul, nos períodos de dois mil e sete, dois mil e oito e dois mil e nove. O instrumento a ser analisado não pôde ser especificamente as avaliações, mas apenas alguns dos itens que as compõem. Alguns dos itens do SAERS compõem as avaliações do Sistema Nacional de Avaliação da Educação Básica (SAEB) que, por sua vez, não disponibiliza nenhum item, ou seja, a avaliação completa do SAERS não é disponibilizada pelo fato supracitado. Subsidiada pelo estudo teórico, realizou-se a análise dos itens, dos quais foram selecionados os que enfatizam ou possuem maior possibilidade de mobilizar os diferentes Registros de Representação Algébricos. A partir desse processo, foram selecionados três, um de cada avaliação analisada, sendo estes os que possuem maior possibilidade de conversão/mobilização dos Registros de Representação Algébricos, ou seja, os itens podem ser resolvidos a partir de conceitos algébricos. A partir dessa seleção, realizouse uma análise dos três itens, dos quais foram identificados os Registros de Representação Algébricos e as possíveis mobilizações, ambos necessários para a solução dos mesmos. Tal análise proporcionou a elaboração deste trabalho, sendo que, sequencialmente, será explicitada uma discussão acerca do Ensino de Álgebra e os Registros de Representação Semiótica, seguida dos Registros de Representação Algébricos e suas transformações. Em seguida, será apresentada a análise dos três itens selecionados que, por sua vez, possuem maior possibilidade de mobilizar os Registros de Representação Algébricos. 


\section{A avaliação do SAERS}

O Sistema de Avaliação do Rendimento Escolar do Rio Grande do Sul (SAERS) é um sistema de avaliação de ordem pública que abrange todas as escolas do estado do Rio Grande do Sul, considerando escolas públicas e privadas ${ }^{1}$. Estas avaliações estão regulamentadas pela Lei de Diretrizes e Bases (LDB), Lei n 9.394/96, art. 9º, inciso VI, que impõe à União "assegurar processo nacional de avaliação do rendimento escolar no ensino fundamental, médio e superior, em colaboração com os sistemas de ensino, objetivando a definição de prioridades e a melhoria da qualidade de ensino" (2009, p. 115). De acordo com os endereços eletrônicos oficiais do SAERS e da Secretaria da Educação, o SAERS é uma iniciativa da Secretaria da Educação com o intuito de verificar as habilidades cognitivas desenvolvidas por alunos, da segunda série e quinta série do Ensino Fundamental, e primeiro ano do Ensino Médio, nas disciplinas de Matemática e Português.

Esses períodos são entendidos como um momento decisivo no percurso escolar dos alunos, de forma a permitir a intervenção pedagógica para a correção dos problemas de aprendizagem detectados no processo de avaliação. Tem, ainda, a finalidade de melhorar a qualidade do ensino e fornecer assistência para a correção das políticas educacionais. Além disso, a avaliação do SAERS complementa as avaliações realizadas pelo Ministério da Educação (Sistema de Avaliação da Educação Básica (SAEB) e da Provinha Brasil), pois estas são aplicadas a alunos da quarta série e oitava série do Ensino Fundamental e terceiro ano do Ensino Médio.

Conforme os Boletins Pedagógicos (2007 e 2008), a realização das avaliações sistemáticas iniciou no Rio Grande do Sul, no ano de mil novecentos e noventa e seis, na qual foram avaliados alunos da segunda série, quinta série e sétima série do Ensino Fundamental e segundo ano do Ensino Médio. Em mil novecentos e noventa e sete e mil novecentos e noventa e oito, aplicaram-se as avaliações de Língua Portuguesa, Redação e Matemática aos alunos de quarta série e oitava série do Ensino Fundamental, e terceiro ano do Ensino Médio.

O quadro abaixo explicitado apresenta o número de alunos que participaram das avaliações, considerando a primeira avaliação do SAERS que se realizou, no ano de dois mil e cinco e, em sequência, as avaliações realizadas nos anos de dois mil e sete, dois mil e oito e a previsão para o ano de dois mil e nove.

\footnotetext{
${ }^{1}$ As escolas municipais e/ou particulares somente participam da avaliação se suas mantenedoras aderirem ao SAERS.
} 
Quadro 1 - Total de alunos que participaram das avaliações do SAERS nos anos de 2005, 2007, 2008 e previsão para 2009, considerando as escolas públicas, particulares e federais.

\begin{tabular}{|c|c|c|c|c|c|}
\hline $\begin{array}{c}\text { Ano de } \\
\text { Realização }\end{array}$ & $\begin{array}{c}\text { Escolas } \\
\text { Estaduais }\end{array}$ & $\begin{array}{c}\text { Escolas } \\
\text { Municipais }\end{array}$ & $\begin{array}{c}\text { Escolas } \\
\text { Particulares }\end{array}$ & $\begin{array}{c}\text { Escolas } \\
\text { Federais }\end{array}$ & $\begin{array}{c}\text { Total de } \\
\text { Alunos }\end{array}$ \\
\hline 2005 & 223 & 1.243 & 0 & 0 & 46.425 \\
\hline 2007 & $\begin{array}{c}\text { Todas as } \\
\text { escolas da } \\
\text { rede estadual }\end{array}$ & $\begin{array}{c}56 \\
\text { municípios }\end{array}$ & 18 & 1 & 288.734 \\
\hline 2008 & 2.690 & 5 municípios & 15 & 1 & 246.891 \\
\hline $\begin{array}{c}2009 \\
\text { previsto) }\end{array}$ & 2.470 & 332 & 28 & 0 & 325.200 \\
\hline
\end{tabular}

Fonte: Dados disponíveis em http://www.saers.caedufjf.net/saers-inst/. Acesso em 03 de novembro de 2009.

As avaliações são elaboradas a partir da Matriz de Referência para Avaliação. A Matriz de Referência para Avaliação é elaborada a partir da Matriz de Referência Curricular do Sistema de Ensino. Ela é composta por descritores que descrevem as habilidades básicas, consideradas importantes para a aprendizagem, a serem desenvolvidas nos domínios: Espaço e Forma, Grandezas e Medidas, Números e Operações/Álgebra, e Funções e Tratamento da Informação, que também compõem os blocos de conteúdos dos Parâmetros Curriculares Nacionais - Matemática, bem como influenciam na elaboração das Matrizes de Referência. Ainda possui como referência uma versão preliminar da Matriz do Sistema Nacional de Avaliação da Educação Básica (SAEB).

As questões das avaliações são chamadas de itens, sendo que cada um deles contempla apenas uma habilidade, ou seja, os itens contemplam todos os domínios e seus descritores. A elaboração destes segue um padrão técnico e pedagógico com o intuito de produzir itens de boa qualidade. Parte dos itens que formam as avaliações são elaborados por professores especialistas nas áreas avaliadas e utilizam as Matrizes de Referência. No entanto, alguns itens compõem o banco de dados das avaliações do SAEB, sendo que estes componentes não são disponibilizados pelo Ministério da Educação. Levando em consideração este fato, somente alguns itens do SAERS são disponibilizados nos Boletins Pedagógicos e endereços eletrônicos para que as escolas e professores os utilizem a fim de aperfeiçoarem a prática docente. Foram disponibilizados nos anos de dois mil e sete, dois mil e oito e dois mil e nove, respectivamente, trinta, onze e dez itens das avaliações aplicadas.

Todos os itens são de múltipla escolha e constituídos pelo enunciado, que apresenta uma situação, pelo comando, que é a pergunta chave ou que solicita ao aluno a resposta certa e as 
alternativas de resposta, compostas por quatro opções (A, B, C e D), na qual apenas uma é correta. É válido ressaltar que alguns dos itens que constituem as avaliações para o primeiro ano do Ensino Médio constituem, também, as avaliações para a quinta série do Ensino Fundamental.

Para a elaboração das avaliações, é contratada uma assessoria técnica especializada na área de elaboração de avaliações, sendo esta responsável por todos os procedimentos, desde a elaboração até a aplicação e correção das avaliações. O processo de aplicação das avaliações ocorre em um período pré-definido pela Secretaria da Educação do Estado do RS em todas as escolas participantes. Durante a aplicação, também ocorre paralelamente a aplicação de questionários aos alunos, professores e equipe diretiva das escolas, nos turnos da manhã, tarde e noite. Para a aplicação das avaliações, são contratados profissionais que trabalharam durante a aplicação das avaliações. Esses passam por um período de treinamento distinto, para coordenadores e supervisores regionais. Os supervisores regionais são responsáveis pelo treinamento dos aplicadores. As avaliações e questionários são mantidos em sigilo, e empacotados em envelopes de plástico lacrados para serem abertos somente no momento da aplicação. São distribuídos aos alunos dois cadernos de avaliação, um de Português e um de Matemática. Após a realização das avaliações, estes são recolhidas e devolvidas às Coordenadorias Regionais de Educação e, posteriormente, à instituição contratada.

Após a aplicação das avaliações, os itens são analisados e avaliados à luz da Matriz de Referência do SAERS, sendo que cada escola é avaliada separadamente. Nesta análise não são analisados somente acertos e erros, mas consideradas todas as respostas, identificando quais as possíveis competências e habilidades atingidas pelo aluno ao colocar determinada resposta. As respostas também recebem um tratamento estatístico que possibilita "medir" a habilidade do aluno. A partir da análise são elaborados os Boletins Pedagógicos de Avaliação da Educação, considerando cada escola individualmente. Estes Boletins são enviados à escola para que esta tenha conhecimento do desempenho dos seus alunos, e para que os professores reflitam a partir da análise detalhada. Isto é, são editados, com o intuito de motivar a escola a melhorar a qualidade dos projetos pedagógicos e a prática docente. Nestes, consta uma parcela dos itens que compuseram as avaliações, juntamente com as respostas dos alunos e uma breve análise, já citada anteriormente.

Os resultados das avaliações são utilizados, ainda, para identificar o rendimento das escolas, a qualidade do ensino, bem como, identificar e auxiliar posteriormente as escolas que possuem 
dificuldades. É possível, também, que sejam comparados os resultados atuais com os resultados anteriores, identificando, assim, a evolução do desempenho dos alunos.

\section{Teoria dos Registros de Representação Semiótica e o Ensino de Álgebra}

O aporte teórico dos Registros de Representação Semiótica possibilita compreender que a Matemática, diferentemente de outras áreas de conhecimento como Física, Química e Biologia, não é perceptível ou observável através de objetos concretos, sendo necessária, para sua apreensão, a utilização e articulação das representações semióticas (DUVAL, 2003).

Para que a apreensão dos objetos matemáticos seja significativa, Duval (2003) propõe a mobilização de ao menos dois Registros de Representação de um mesmo objeto matemático por parte do aluno, ou seja, considerando, por exemplo, a possibilidade de representar uma função através de uma equação, tabela ou gráfico, e mobilizá-los em ambos os sentidos. Damm (1999) afirma que as Representações Semióticas são necessárias para as atividades cognitivas do pensamento, ou seja, sem as Representações Semióticas não é possível que o aluno tenha uma compreensão conceitual significativa. É preciso considerar, ainda, os dois tipos de transformação propostos por Duval (2003): o Tratamento e a Conversão.

O Tratamento dos registros consiste em permanecer em um único registro do objeto matemático, realizando, por exemplo, somente a substituição da letra por um número na equação $3 x+6=15$. A Conversão, no entanto, consiste em mudar de registro dentro de um único objeto matemático, ou seja, "a conversão se dá entre os registros diferentes" (DAMM, 1999, p. 146). Como exemplo, pode-se considerar o objeto matemático função e sua conversão para o registro gráfico ou equação, em ambos sentidos. É importante levar em conta que existe maior dificuldade por parte dos alunos ao realizar a conversão entre os registros, pois exige uma compreensão significativa dos conceitos matemáticos e o estabelecimento de diferenças entre as representações.

Para a compreensão de conceitos algébricos, a Teoria dos Registros de Representação Semiótica também assume papel muito importante. Coxford e Shulte (1995), assim como os Parâmetros Curriculares Nacionais - Matemática (1998), afirmam que o aluno precisa ter uma base do que é a Álgebra para dar significado e compreender os diferentes conceitos algébricos, ou seja, compreender as diferentes Dimensões da Álgebra, propostas nos Parâmetros Curriculares. 
Este estudo teórico possibilitou a identificação dos Registros de Representação Algébricos, essenciais para a apreensão de conceitos algébricos, e de grande valia se utilizados para subsidiar a elaboração de itens, utilizados para avaliar as competências e habilidades desenvolvidas pelos alunos. Pode-se destacar os seguintes Registros Algébricos: Registro Aritmético (RA), expressa ou traduz padrões numéricos e geométricos a partir de uma linguagem algébrica; Registro Funcional (RF), a letra assume papel de variável, expressando relações; Registro de Equações (REq), as letras assumem o papel de incógnitas; Registro Estrutural (RE), as letras são entendidas como símbolos abstratos; Registro da Língua Materna (RLM), consiste em situações apresentadas na língua portuguesa; Registro Figural (RFig), compreende figuras que não assumem apenas o papel de ilustrar uma situação e figuras geométricas; Registro Gráfico $(\mathrm{RG})$, compreende a utilização de gráficos no plano cartesiano; e o Registro Numérico ( $\mathrm{RN})$, que compreende sequências numéricas e tabelas.

Ao considerar a conversão entre estes registros, será possibilitado ao aluno compreender de fato os conceitos algébricos e reconhecer as diferentes formas de representar uma expressão algébrica, adquirindo uma base concreta para uma aprendizagem de Álgebra mais sólida e rica em significados (BRASIL, 1998). O Referencial Curricular do Rio Grande do Sul (RS, 2009) destaca que é importante ao aluno construir o conhecimento matemático por meio do desenvolvimento de competências e habilidades, enfatizando a resolução de problemas, onde se pode considerar a mobilização dos Registros de Representação Algébricos.

Este estudo teórico, bem como a identificação dos Registros de Representação Algébricos, possibilitou a análise de parte dos itens das avaliações dos SAERS para o primeiro ano do Ensino Médio, bem como, posteriormente, seleção e análise de três itens, explicitados a seguir.

\section{Proposições dos Itens}

Considerando os Registros de Representação Algébricos, foi possível analisar os itens que compõem as avaliações do SAERS para os alunos do primeiro ano do Ensino Médio. A partir desta análise, foram identificados três itens que possuem maior possibilidade de mobilização dos Registros Algébricos. Cada item selecionado encontra-se em uma das três avaliações analisadas. Dentre os trinta itens disponibilizados da avaliação de dois mil e sete, quatorze apresentam possibilidades de mobilizar os $\mathrm{RR}^{2}$ Algébricos. No ano de dois mil e oito

\footnotetext{
${ }^{2}$ A partir de agora, ao dar referência aos Registros de Representação, será utilizado o termo "RR".
} 
identificou-se a possibilidade de mobilização em dois itens, dos onze disponíveis. Em dois mil e nove, de dez itens disponíveis apenas três têm a possibilidade de mobilizar os RR Algébricos.

O item abaixo indicado (Figura 1) é proposto na avaliação do ano de dois mil e sete.

Figura 1 - Item proposto na avaliação de Matemática do SAERS no ano de 2007.

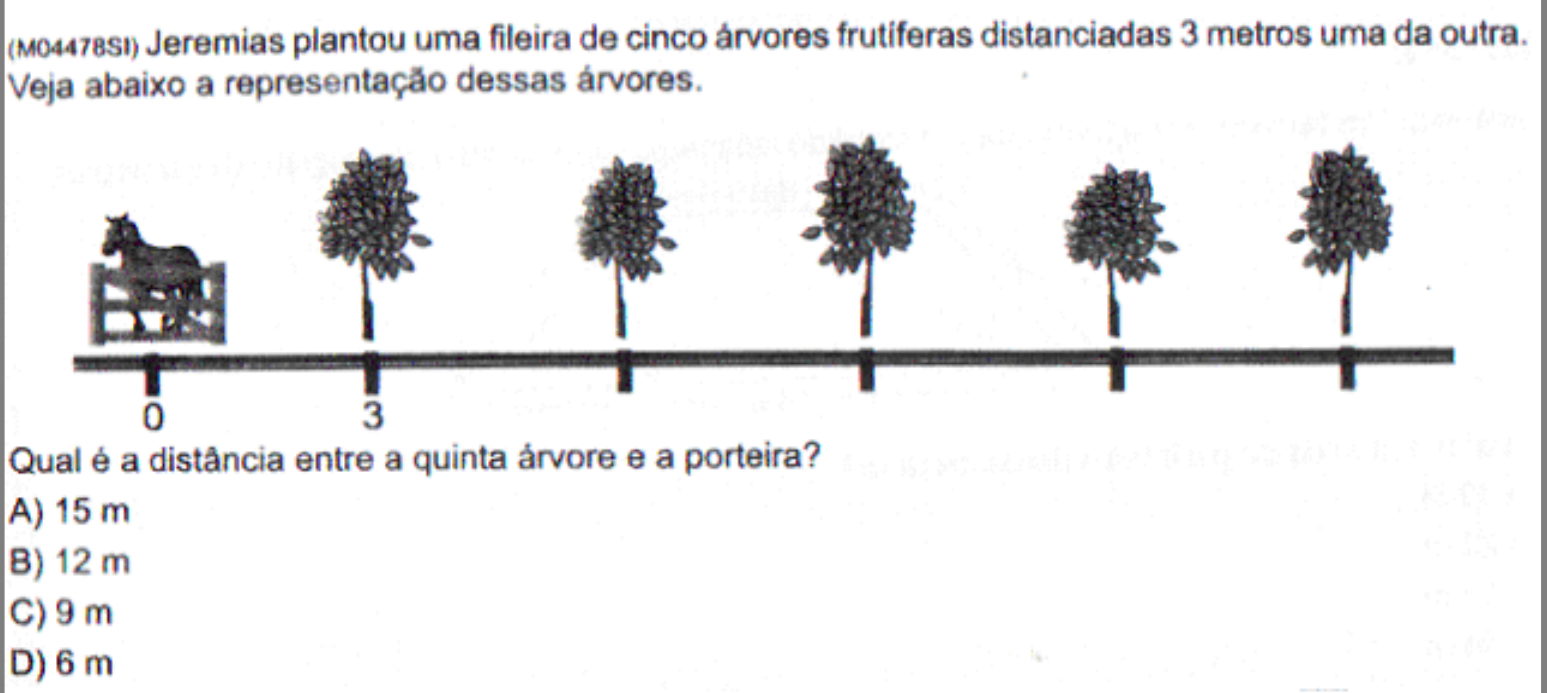

Fonte: Boletim Pedagógico de Matemática $-1^{\circ}$ ano de Ensino Médio, 2007, p. 40.

Conforme o Boletim Pedagógico de Avaliação da Educação, neste item o aluno precisa desenvolver a habilidade de resolver problemas, realizando apenas diferentes operações com números inteiros. Com este olhar para o item, é possível identificar o Registro da Língua Materna, o Registro Figural e o Registro Numérico. O Registro da Língua Materna está explícito no enunciado do item, onde se faz necessária a identificação de que a cada três metros uma árvore é plantada. O Registro Figural é expresso através da figura que, neste caso, não assume apenas o papel de ilustrar a situação, mas auxilia o aluno a visualizar a situação, ou utilizá-la como uma reta numérica, na qual realizará o Registro Numérico podendo, também, realizar a multiplicação do número solicitado de árvores pela distância de três metros. Identificando, portanto, apenas um Tratamento Numérico do item, sem envolver Registros Algébricos de fato.

Com um olhar algébrico, este item poderia ser proposto de forma a utilizar a Álgebra para resolver o problema, considerando a mobilização dos Registros Algébricos, pois a Álgebra 
possibilita aos alunos realizar abstrações e generalizações, condições básicas para o Ensino de Matemática no Ensino Médio.

A análise deste item possibilitou detectar que não é dada ênfase aos Registros Algébricos e suas mobilizações. No entanto, se este fosse estruturado de forma diferenciada, facilitaria a conversão do Registro da Língua Materna e do Registro Figural para os Registros Funcionais de Equações e Gráfico, marcando a ideia de mobilidade dos registros, ajudando a desencadear a aprendizagem, levando em conta que o aprendizado de Matemática acontece com a mobilização de, pelo menos, dois RR de um mesmo objeto matemático (DUVAL, 2003).

Figura 2 - Item proposto na avaliação de Matemática do SAERS no ano de 2008.

(M090147A8) Rose multiplicou a idade atual de seu filho pela idade que ele terá daqui a 5 anos e obteve como resultado 14 anos.

Qual é a idade atual do filho de Rose?

A) 2 anos.

B) 5 anos.

C) 7 anos.

D) 9 anos.

Fonte: Boletim Pedagógico de Matemática - $1^{\circ}$ ano de Ensino Médio, 2008, p. 70.

Este item tem como objetivo desenvolver no aluno suas habilidades em relação aos conceitos algébricos, quando entende-se que ocorreu uma pequena evolução em relação a esta concepção, considerando o item apresentado anteriormente (Figura 1). Este item propõe a possibilidade de exploração da situação problema, a partir dos Registros da Língua Materna e de Equações. O Registro da Língua Materna, explícito no enunciado, exige que o aluno compreenda o proposto. Para que o aluno realize a mobilização do Registro da Língua Materna para o Registro de Equação, que é a solução do item, é necessário que o aluno reconheça a idade atual do filho de Rose como uma incógnita $(x)$. Em seguida, que esta idade, multiplicada pela idade do filho de Rose em cinco anos, terá como resultado o número quatorze, sendo possível registrar a equação $x \cdot(x+5)=14$. Ou seja, obtendo um pensamento algébrico, identificando o papel da letra como incógnita em uma equação. A partir da identificação deste registro, o aluno alcança uma Equação de segundo grau onde, resolvendoa, atingirá dois valores distintos para $x$, sendo estes $x^{\prime}=2$ e $x^{\prime \prime}=-7$. Neste momento, o aluno terá que identificar qual o valor que melhor se aplica a esta situação problema, identificando, portanto, que o primeiro valor é o correto, pois não existe idade negativa. 
A partir da análise realizada, identifica-se a mobilização do Registro da Língua Materna para o Registro de Equações, fazendo com que o aluno desenvolva habilidades não somente para realizar operações com números naturais, mas desenvolva habilidades que exijam uma compreensão conceitual acerca dos conceitos algébricos.

Figura 3 - Item proposto na avaliação de Matemática do SAERS no ano de 2009.

(M090485A9) Numa papelaria, cada caderno custa 5 reais a mais que um jogo de canetas. Marcos comprou 4 cadernos e 3 jogos de canetas e pagou por essa compra 106 reais.

Qual é a equação que expressa esse problema?
A) $3(x+5)+4 x=106$
B) $4(x+5)+3 x=106$
C) $4 x+3 x+5=106$
D) $4 x-5+3 x=106$

Fonte: Disponibilizado pela Equipe de Coordenação de elaboração da avaliação do SAERS em 17 de maio de 2010.

Esta situação foi disponibilizada para esta pesquisa pela Equipe de Coordenação de elaboração da avaliação, pois o Boletim Pedagógico de Avaliação da Educação deste período ainda não está disponível.

A solução deste item também exige que o aluno tenha um pensamento algébrico, porém de forma mais explícita, pois apresenta incógnitas em sua estrutura, diferente dos itens supracitados. Este item envolve dois Registros de Representação Algébricos: o Registro da Língua Materna e o Registro de Equações. Identifica-se como Registro da Língua Materna no enunciado do item, sendo necessário ao aluno compreender o proposto, tendo um pensamento algébrico para, então, realizar a conversão deste registro para o Registro de Equações que, por sua vez, é a solução do item. Para tanto, o aluno precisa identificar como $x$, ou seja, uma incógnita, o número de jogos de canetas. É preciso identificar, também, que o número de cadernos é igual ao número de jogos de canetas mais cinco $(x+5)$. Considerando as informações do Registro da Língua Materna, é possível ao aluno registrar a equação $4 \cdot(x+5)+3 \cdot x=106$, ou seja, realizar o Registro de Equações.

A partir desta análise, identifica-se a conversão do Registro da Língua Materna para o Registro de Equações, exigindo do aluno uma interpretação mais aprofundada do Registro da Língua Materna, bem como dos conceitos algébricos. Considerando a importância de o aluno 
compreender significativamente os conceitos algébricos, vale ressaltar a importância de envolver não somente o Registro da Língua Materna e o Registro de Equações, mas envolver registros que vão além de a letra assumir apenas o papel de incógnita.

\section{Considerações Finais}

Esta pesquisa teve por objetivo analisar os itens das avaliações de Matemática do SAERS, aplicadas a alunos do primeiro ano do Ensino Médio nos períodos de dois mil e sete, dois mil e oito, e dois mil e nove, buscando discutir e identificar as possibilidades de exploração dos itens, considerando os conceitos algébricos e a Teoria dos Registros de Representação Semiótica.

Para atingir tal objetivo - definido por meio do estudo e análise dos Parâmetros Curriculares Nacionais - Matemática, da Matriz de Referência do SAERS, do Referencial Curricular do Rio Grande do Sul e por fim dos itens das avaliações - buscou-se subsídio na Teoria dos Registros de Representação Semiótica de Duval (2003), definindo alguns critérios com o intuito de avaliar se os itens possibilitam a mobilização e coordenação dos Registros de Representação Algébricos.

A partir do estudo e análise desenvolvidos, foi possível identificar que existe grande ênfase em relação às situações problema, considerando, em grande parte, apenas um tratamento numérico através das diferentes operações. Ou seja, as análises possibilitaram identificar que grande parte dos itens abordam situações do cotidiano dos alunos, a partir de situações problema. Tais situações são propostas apenas para avaliar a habilidade do aluno em resolver as cisrcunstâncias sugeridas com números naturais, envolvendo os diferentes significados das operações (adição, subtração, multiplicação, divisão e potenciação). Não é dada ênfase à mobilização dos registros, sendo que uma das poucas possibilidades ressaltadas nos itens é a conversão do Registro da Língua Materna para o Registro de Equações.

Poderia haver maior ênfase dos Registros Algébricos nos itens disponíveis, considerando toda a caminhada conceitual algébrica que o aluno obteve até este período de ensino, com ênfase para a sétima série e oitava série do Ensino Fundamental. Entende-se, que é importante ser avaliado nos alunos não somente a habilidade de somar, subtrair, multiplicar ou dividir números inteiros, decimais, racionais ou fracionários. É imprescindível que seja avaliada a compreensão dos conceitos, especificamente de Álgebra, e a capacidade de mudar de registro, através da compreensão conceitual e das diferentes formas de representar um único objeto 
matemático. Considerando tal fato, existem garantias de que realmente houve um aprendizado e uma compreensão conceitual significativos por parte dos alunos, onde a Álgebra não é entendida apenas como a troca de letras por números, mas como o estabelecimento de procedimentos e relações expressos numa forma simplificada geral (Coxford e Shulte, 1995).

Como as questões disponíveis tem a finalidade de orientar o professor, seria pertinente que esta circunstância fosse considerada. Estas situações permitem afirmar, juntamente com Coxford e Shulte, que

[...] de um lado há uma necessidade urgente de novos instrumentos de aferição, que possam avaliar adequadamente os níveis superiores do raciocínio e a capacidade de resolver problemas. De outro, há o perigo permanente de responder às exigências de notas mais altas, enfraquecendo o processo de avaliação mais ainda ou "ensinando para o exame", enfatizando apenas as habilidades mecânicas que são fáceis de medir. (1995, p. 7)

Esta afirmação nos faz refletir acerca da contribuição das avaliações para o desenvolvimento do ensino e, se essa é a forma correta de avaliar as habilidades e competências dos alunos. É necessário considerar que os alunos do Ensino Médio já possuem extensa caminhada em relação ao estudo de conceitos algébricos, pois estes são trabalhados no Ensino Fundamental e reforçados no Ensino Médio, especificamente no primeiro ano. É importante que ocorra um avanço na utilização dos conceitos algébricos em relação aos conceitos aritméticos, pois a Álgebra exige do aluno um pensamento generalizável e abstrato, sendo necessário, para tanto, basear-se teoricamente nos Registros de Representação, consequentemente ocorrendo avanço necessário para o Ensino de Matemática no Ensino Médio.

Acredita-se que, se a Teoria dos Registros de Representação Semiótica, bem como a articulação entre os registros, for considerada, com ênfase no momento de elaborar e analisar os itens que compõe as avaliações, e isto refletido nos planos de ensino, será possível afirmar que haverá uma compreensão conceitual significativa por parte dos alunos, levando em conta que a compreensão em Matemática, especificamente no Ensino de Álgebra, implica a capacidade de mudança de registro (DUVAL, 2003). 


\section{Referências}

ALMOULOUD, Saddo Ag. Fundamentos da didática da matemática. Curitiba, UFPR, 2007.

BRASIL. Parâmetros Curriculares Nacionais: Matemática. Secretaria de Educação. Brasília: MEC, SEF, 1998. (Anos Finais do Ensino Fundamental)

BREUNIG, Raquel Taís; NEHRING, Cátia Maria; POZZOBON, Marta Cristina Cezar. Registros de Representação e o Ensino de Álgebra: conversões realizadas por alunos da oitava série em uma situação de ensino. In: JORNADA NACIONAL DE EDUCAÇÃO MATEMÁtICA, 3. 2010, Passo Fundo. Anais... Passo Fundo: Ed. Universidade de Passo Fundo, 2010. P. 1-18.

COXFORD, Arthur F.; SHULTE, Albert P. As idéias da álgebra. Traduzido por Hygino H. Domingues. São Paulo: Atual, 1995.

DAMM, Regina Flemming. Registros de Representação. In: MACHADO, Sílvia Dias Alcântara. et al. Educação Matemática: uma introdução. São Paulo: EDUC, 1999. P. 135153.

DUVAL, Raymond. Registros de Representações Semióticas e Funcionamento Cognitivo da Compreensão em Matemática. In: MACHADO, Sílvia Dias Alcântara. (Org). Aprendizagem em matemática: registros de representação semiótica. Campinas, SP: Papirus, 2003. p. 1133.

Equipe de Coordenação do Sistema de Avaliação do Rendimento Escolar do Rio Grande do Sul. Juiz de Fora, 2009.

LEI No 9.394, de 20 de dezembro de 1996. In: PINTO, T. B. et al. (Org). Políticas, estrutura e gestão da educação básica. Ijuí, RS: Unijuí, 2009. P.113-136.

RIO GRANDE DO SUL. Referencial Curricular: Matemática e suas tecnologias. Secretaria de Estado da Educação. 2009.

RIO GRANDE DO SUL. Boletim Pedagógico de Avaliação da Educação: SAERS 2007. Secretaria de Estado da Educação. Universidade Federal de Juiz de Fora, Faculdade de Educação, CAEd. v. 1 (jan./dez. 2007). Juiz de Fora, 2007. (Matemática $1^{\text {o }}$ ano do Ensino Médio)

RIO GRANDE DO SUL. Boletim Pedagógico de Avaliação da Educação: SAERS 2008. Secretaria de Estado da Educação. Universidade Federal de Juiz de Fora, Faculdade de Educação, CAEd. v. 1 (jan./dez. 2008). Juiz de Fora, 2008. (Matemática $1^{\circ}$ ano do Ensino Médio)

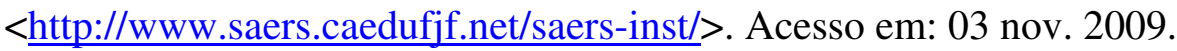

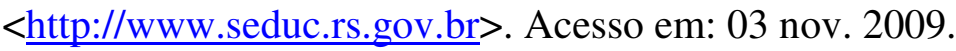

